\title{
Applications of Microscopy to Advanced Magnetic Data Storage Materials
}

\author{
A.K. Petford-Long*, B Warot*, A Cerezo*, Y-G Wang*, D J Larson**, Y Q Ma*, M T Kief**, J \\ Nickel*** \\ *Dept of Materials, University of Oxford, Parks Road, Oxford OX1 3PH, UK \\ **Seagate Technology, 1 Disc Drive, Bloomington, MN 55435, USA. \\ *** Hewlett-Packard Labs, 1501 Page Mill Road, Palo Alto, CA 94304, USA.
}

The trends in data storage towards higher storage density, and towards portable storage solutions, have led to the need to develop suitable materials. Many of these consist of stacks of nanometrethick layers, with microstructure that is inhomogeneous on a fine scale. Examples of such materials are spin-valve (SV) and spin tunnel junction (STJ) structures which rely on the giant magnetoresistance (GMR) effect [1] and have applications as magnetoresistive read-heads [2] and as memory elements [3]. Another example is arrays of magnetic dots or antidots [4] which are being developed for potential use as patterned magnetic media, with each dot (or magnetic region between adjacent antidots) supporting a magnetic bit. In their simplest form, SV and STJ structures consist of a ferromagnetic (FM) layer whose magnetisation direction is fixed by an adjacent antiferromagnetic (AF) pinning layer through exchange coupling. The pinned FM layer is separated by a nonmagnetic spacer layer from a second FM layer (sense layer) whose magnetisation can be rotated in a low external magnetic field. The spacer is a metal in an SV and an insulator in an STJ.

The microstructure and chemical profile across the layers are critical in determining the magnetic and transport properties of the devices, and therefore need to be critically controlled. In addition, the films currently being developed for device applications are mainly polycrystalline, which makes it more complicated to disentangle the effects of different microstructural parameters than would be the case for single crystal epitaxial films. We have applied HREM, TEM chemical mapping, Lorentz TEM (LTEM ) and three-dimensional atom probe (3DAP) analysis [5] to information storage materials and this paper will present some of these results. For example, Figure 1 shows a plot of MR versus anneal time and temperature for a series of STJs with the same nominal structure. HREM images of films annealed at $280^{\circ} \mathrm{C}$ show that after a $0.5 \mathrm{hr}$ anneal the tunnel barrier is still amorphous (left-hand image), where after a $3 \mathrm{hr}$ anneal crystalline regions are present in the barrier (right-hand image). In addition to this change, TEM chemical mapping has indicated an increase in Mn diffusion through the structure, and these changes have led to a reduction in MR.

The behaviour of devices made from these materials also relies on the local magnetic domain structure and magnetisation reversal mechanism. One of the techniques enabling micromagnetic studies at the sub-micron scale is Lorentz transmission electron microscopy (LTEM) which allows the magnetic domain structure and magnetisation reversal mechanism of a FM material to be investigated dynamically with a resolution of a few nanometres. An example of this type of analysis is shown in Figure 2. The Foucault mode LTEM image shows an array of STJ elements in which the contrast indicates the relative alignment of the sense and pinned FM layers. As can be seen from the associated plot, the reversal field for the sense layer is a function of the aspect ratio of the STJ element and also of the width of the element, with the difference in aspect ratio dependence being a result of the relative importance of the energy terms that contribute to the reversal field. 
[1] R L White, IEEE Trans. Mag. 30(2) (1994) 346.

[2] J A Brug, T C Anthony and J H Nickel, MRS Bulletin 21 (1996) 23.

[3] J M Daughton, Thin Solid Films 216 (1992) 162.

[4] R P Cowburn, A Adeyeye and J A C Bland, J. Magn. Magn. Mater. 173 (1997) 193.

[5] M K Miller, A Cerezo, M G Hetherington \& G D W Smith, 'Atom probe field ion microscopy', (Oxford University Press, Oxford, 1996).

[6] This research was supported by the Engineering and Physical Sciences Research Council, Hewlett-Packard Labs, Palo Alto and Seagate Technology, Minneapolis and Springtown, N.I..
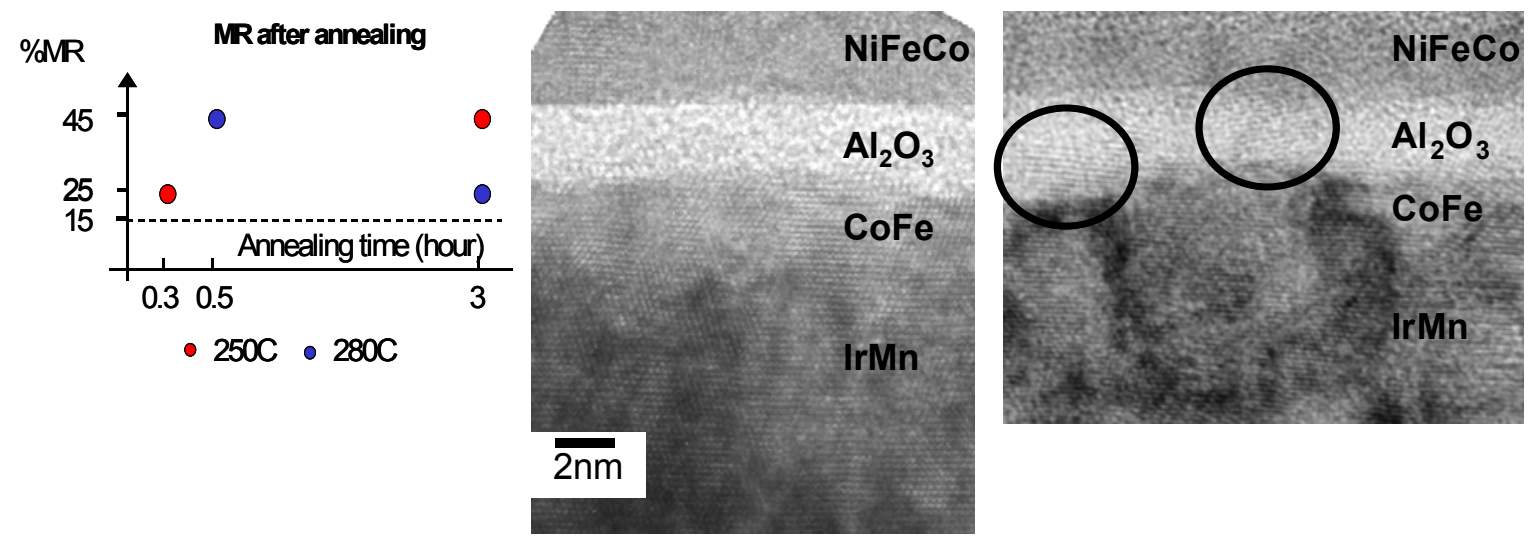

Figure 1. MR ratios for TMR structures annealed at $250^{\circ} \mathrm{C}$ or $280^{\circ} \mathrm{C}$ for different periods of time. The HREM images are of the films annealed at $280^{\circ} \mathrm{C}$ for $0.5 \mathrm{hr}$ (left-hand image) and 3 $\mathrm{hr}$ (right-hand image). Note crystalline regions in barrier after $3 \mathrm{hr}$ anneal.
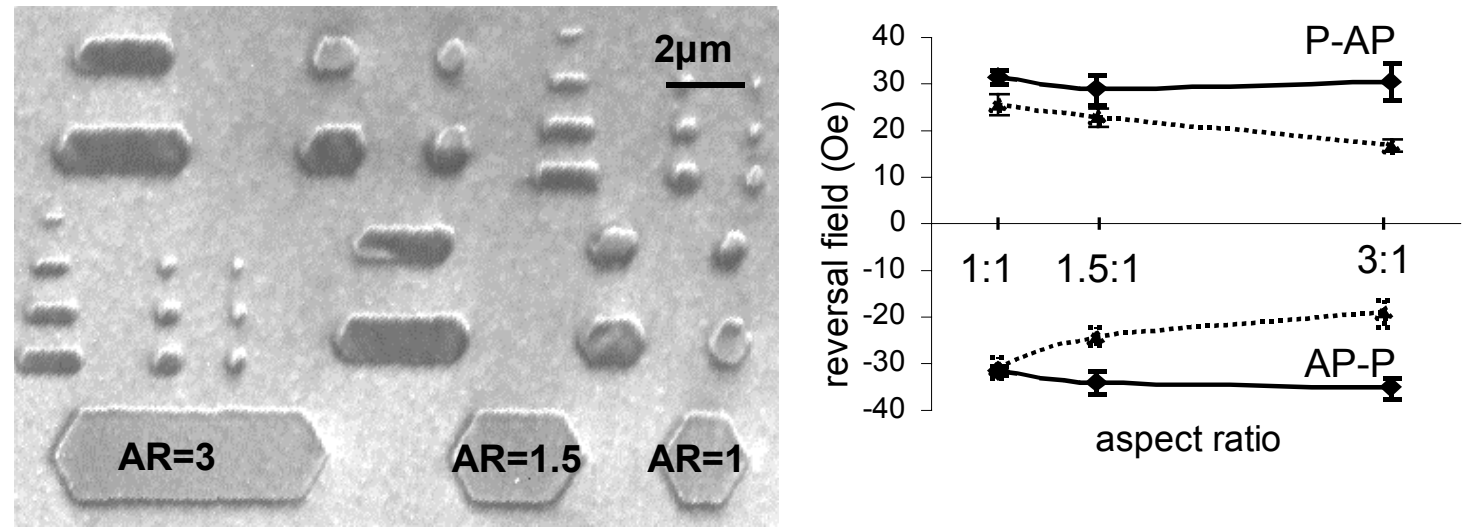

Figure 2 Foucault mode LTEM images of an array of STJ structures. Dark contrast indicates parallel alignment of the magnetisation in the sense and pinned FM layers, bright contrast indicates antiparallel alignment. The plot shows the reversal fields for the parallel-to-antiparallel (P-AP), and antiparallel-to-parallel (AP-P) reversal of the sense layer magnetisation for $2 \mu \mathrm{m}$ wide (dashed line) and $0.7 \mu \mathrm{m}$ wide (solid line) elements as a function of aspect ratio (AR). 